\title{
Global qualitative analysis of a ratio-dependent predator-prey system
}

\author{
Yang Kuang ${ }^{1, \star}$, Edoardo Beretta ${ }^{2, \star \star}$ \\ ${ }^{1}$ Department of Mathematics, Arizona State University, Tempe, AZ 85287, USA \\ ${ }^{2}$ Istituto di Biomatematica, Universita di Urbino, I-61029 Urbino, Italy
}

Received 5 April 1997; received in revised form 14 July 1997

\begin{abstract}
Ratio-dependent predator-prey models are favored by many animal ecologists recently as more suitable ones for predator-prey interactions where predation involves searching process. However, such models are not well studied in the sense that most results are local stability related. In this paper, we consider the global behaviors of solutions of a ratio-dependent predator-prey systems. Specifically, we shall show that ratio dependent predator-prey models are rich in boundary dynamics, and most importantly, we shall show that if the positive steady state of the so-called Michaelis-Menten ratio-dependent predator-prey system is locally asymptotically stable, then the system has no nontrivial positive periodic solutions. We also give sufficient conditions for each of the possible three steady states to be globally asymptotically stable. We note that for ratio-dependent systems, in general, local asymptotic stability of the positive steady state does not even guarantee the so-called persistence of the system, and therefore does not imply global asymptotic stability. To show that the system has no nontrivial positive periodic solutions. we employ the so-called divergency criterion for the stability of limit cycles in planar systems and some critical transformations.
\end{abstract}

Key words: Global stability - Predator-prey system - Limit cycles Liapunov function - Dulac criterion

\section{Introduction}

The dynamic relationship between predators and their prey has long been and will continue to be one of the dominant themes in both ecology and

\footnotetext{
${ }^{\star}$ Research partially supported by NSF Grant DMS-9306239.

${ }^{\star \star}$ Research supported by "Gruppo Nazionale per la Fisica Matematica", C.N.R., Italy. AMS Subject Classification (1991): 34C05, 34C25, 92A15.
} 
mathematical ecology due to its universal existence and importance (Berryman (1992)). These problems may appear to be simple mathematically at first sight, they are, in fact, often very challenging and complicated. Although the predator-prey theory has seen much progress in the last forty years, many long standing mathematical and ecological problems remain open (Kuang and Freedman (1988), Kuang (1988, 1990), Berryman (1992), Beretta and Kuang (1996)).

A milestone progress in the study of predator-prey interactions was the discovery of the now well known "paradox of enrichment" by Hairston et al (1960) and by Rosenzweig (1969), which states that according to the Lotka-Volterra type predator-prey theory (with Michaelis-Menten-Holling type functional response) enriching a predator-prey system will cause an increase in the equilibrium density of the predator but not in that of the prey, and will destabilize the community equilibrium. Another similar paradox is the so-called "biological control paradox", which was recently brought into discussion by Luck (1990), stating that according to the classic predator-prey theory, you can not have both a low and stable prey equilibrium density. Both these two paradoxes can be easily illustrated by a standard local stability analysis for the following well studied Lotka-Volterra type predator-prey model with Michaelis-Menten (or Michaelis-Menten-Holling) type functional response (Freedman (1980), May (1974), Maynard Smith (1974))

$$
\left\{\begin{array}{l}
\frac{d x}{d t}=x^{\prime}=a x(1-x / K)-c x y /(m+x) \\
\frac{d y}{d t}=y^{\prime}=y(-d+f x /(m+x))
\end{array}\right.
$$

where $x, y$ stands for prey and predator density, respectively. $a, K, c, m, f, d$ are positive constants that stand for prey intrinsic growth rate, carrying capacity, capturing rate, half capturing saturation constant, conversion rate, predator death rate, respectively. While many of the mathematicians working in mathematical biology may regard these as important contributions that mathematics had for ecology, they are very controversial among ecologists up to this day. Indeed, some ecologist may simply view it as a problem (Arditi and Ginzburg (1989), Arditi and Berryman (1991), Arditi and Saiah (1992), Berryman (1992), Getz (1984), Hanski (1991)). Indeed, the above predictions are simply not in line with many field observations (Arditi and Ginzburg (1989), Arditi et al. (1991), Ginzburg and Akcakaya (1992)).

The controversial part is, of course, not the mathematical analyses of the model, rather, it is the model itself. After all, the classical models are mostly variations of the Lotka (a physical chemist)-Volterra (a mathematician) model which is the product of chemistry, physics and mathematics. Specifically, it is the product of the (chemistry) principle of mass action, (physics) laws of conservation, and (mathematics) elemental differential equations. The missing link is, obviously, the biology content. We simply should not equal or 
approximate all biological interactions by chemical reactions. Recently, there is a growing explicit biological and physiological evidences (Arditi and Saiah (1992), Arditi et al. (1991), Gutierrez (1992)) that in many situations, especially when predators have to search for food (and therefore have to share or compete for food), a more suitable general predator-prey theory should be based on the so-called ratio-dependent theory, which can be roughly stated as that the per capita predator growth rate should be a function of the ratio of prey to predator abundance, and so should be the so-called predator functional responses (see below). This is strongly supported by numerous field and laboratory experiments and observations (Arditi and Ginzburg (1989), Arditi et al. (1991), Hanski (1991)). Generally, a ratio-dependent predator-prey model takes the form

$$
\left\{\begin{array}{l}
x^{\prime}=x f(x)-y p(x / y) \\
y^{\prime}=c y q(x / y)-d y
\end{array}\right.
$$

while the traditional (or prey-dependent) model takes the form

$$
\left\{\begin{array}{l}
x^{\prime}=x f(x)-y p(x) \\
y^{\prime}=c y q(x)-d y
\end{array}\right.
$$

Here $p(x)$ is the so-called predator functional responses. Often, $q(x)$ is replaced by $p(x)$, in which case $c$ becomes the conversion rate. $p(x), q(x)$ satisfy the usual properties such as being nonnegative and increasing, and equal to zero at zero.

Geometrically, the differences of prey-dependent and ratio-dependent models are obvious, the former has a vertical predator isocline, while the latter has a slanted one. There are even more differences in their prey isoclines. Local stability analyses and simulations (Arditi and Ginzburg (1989), Berryman (1992)) show that the ratio-dependent models are capable of producing richer and more reasonable or acceptable dynamics. It is easy to see that the paradox of biological control is no longer valid for ratio-dependent systems. It can also be easily shown that the ratio-dependent type models do not produce the so-called paradox of enrichment.

Since the traditional Lotka-Volterra type predator-prey model with Michaelis-Menten type functional response received great attention among theoretical and mathematical biologists, we will focus our attention here on the ratio-dependent type predator-prey model with Michaelis-Menten type functional response, which takes the form of

$$
\left\{\begin{array}{l}
x^{\prime}=a x(1-x / K)-c x y /(m y+x) \equiv F(x, y) \\
y^{\prime}=y(-d+f x /(m y+x)) \equiv G(x, y) .
\end{array}\right.
$$

In the rest of this paper, we assume that $F(0,0)=G(0,0)=0$. With this assumption, we see that both $F(x, y), G(x, y)$ are continuous on the positive cone $R_{+}^{2}=\{(x, y): x \geqq 0, y \geqq 0\}$. Also, in the rest of this paper, we shall 
assume that the initial condition for the above system satisfies $x(0)>0, y(0)>0$. In the following we say an equilibrium of the above system is globally (asymptotically) stable if it attracts all positive solutions of the system.

The objective of this paper is to perform a global qualitative study on system (1.4). In particular, we shall systematically study the boundary dynamics of system (1.4) and show that if the positive steady state of the system (1.4) is locally asymptotically stable, then the system can not have nontrivial positive periodic solutions. Our method involves a nontrivial application of the socalled divergency criterion for the stability of periodic solutions in planar systems (see Hale (1980), Lemma 3.1 on p. 224) and some critical transformations. This is the same method elegantly applied by Butler et al. (1983) on a chemostat model. We will also present some other related qualitative results for system (1.4).

This paper is organized as follows: in the next section, we present results on the boundedness of solutions (or dissipativity of the systems), permanence results and global stability of $(K, 0)$ and $(0,0)$. Section 3 contains the main result, namely, local asymptotic stability of the positive steady state implies nonexistence of nontrivial positive periodic solutions. It also contains some results on the global stability of the positive steady state. The paper ends with a discussion section containing both simulation work and questions.

\section{Preliminaries, rich boundary dynamics}

In this section, we shall present some preliminary results, including the boundedness of solutions, permanence, and boundary dynamics (the global stability of boundary equilibria $(K, 0)$ and $(0,0))$ for system $(1.4)$. We shall point out here that although $(0,0)$ is defined for system (1.4), it cannot be linearized at. So, local stability of $(0,0)$ can not be studied. Indeed, this singularity at the origin, while causes much difficulty in our analysis of the system, contributes significantly to the richness of dynamics of the model. We also would like point out that some of the results in this section are modified from similar ones in Beretta and Kuang (1997).

For simplicity, we set $b=a / K$ in system (1.4). This results in

$$
\left\{\begin{array}{l}
x^{\prime}=x(a-b x)-c x y /(m y+x), \\
y^{\prime}=y(-d+f x /(m y+x)) .
\end{array}\right.
$$

System (2.1) always has equilibria $(0,0),(K, 0)$ and has a unique positive equilibrium $E^{*}=\left(x^{*}, y^{*}\right)$ if and only if any one of the following two conditions is true:

(i) $d<f<d c /(c-m a)$, when $c>m a$,

(ii) $f>d$, when $c \leqq m a$.

In both cases, we have $x^{*}=[c d-f(c-m a)] /(b m f), y^{*}=x^{*}(f-d) /(d m)$. 
The Jacobian matrix $J_{K}=J(K, 0)$ of system $(1.4)$ at $(K, 0)$ takes the form of

$$
\left(\begin{array}{cc}
-a & -c \\
0 & f-d
\end{array}\right)
$$

Clearly, whenever the positive steady state $E^{*}$ exists, $(K, 0)$ is unstable.

The Jacobian matrix $J^{*}=J\left(x^{*}, y^{*}\right)$ of system (1.4) at $E^{*}$ takes the form of

$$
\left(\begin{array}{cc}
-b x^{*}+\frac{c y^{*} x^{*}}{\left(m y^{*}+x^{*}\right)^{2}} & -\frac{c\left(x^{*}\right)^{2}}{\left(m y^{*}+x^{*}\right)^{2}} \\
\frac{f m\left(y^{*}\right)^{2}}{\left(m y^{*}+x^{*}\right)^{2}} & -\frac{f m x^{*} y^{*}}{\left(m y^{*}+x^{*}\right)^{2}}
\end{array}\right) .
$$

It is easy to see that the determinant of $J^{*}$ is always positive and its trace is

$$
\operatorname{tr}\left(x^{*}, y^{*}\right)=-b x^{*}+(c-f m) \frac{x^{*} y^{*}}{\left(m y^{*}+x^{*}\right)^{2}} .
$$

Clearly, $E^{*}$ is locally asymptotically stable if the trace is less than zero. If we let

$$
r=d / f,
$$

then, after some straightforward computation, we obtain

$$
\operatorname{tr}\left(x^{*}, y^{*}\right)=-a+(1-r)[c+r(c-f m)] / m .
$$

This shows that if $f$ is close to $d$, then $E^{*}$ is locally asymptotically stable.

Standard and simple arguments show that solutions of system (1.4) always exist and stay positive. Indeed, it is obvious that for system (1.4), we have

and

$$
\lim _{t \rightarrow \infty} \sup x(t) \leqq K
$$

$$
y(t)<y(0) \mathrm{e}^{(f-d) t} .
$$

We consider first the boundedness of $y(t)$ for system (1.4). Clearly, if $d \geqq f$, then $\lim _{t \rightarrow \infty} y(t)=0$. So we assume below that $f>d$. We shall show that

Theorem 2.1. For system (1.4), if $f>d$, then

$$
\lim _{t \rightarrow \infty} \sup y(t) \leqq \frac{f-d}{d m} K
$$

Proof. Observe that there exists a $T>0$ such that, for $t>T, x(t)<K$ (otherwise, from the prey equation and the fact that $-d y \leqq y^{\prime} \leqq(f-d) y$, we have $\lim _{t \rightarrow \infty} x(t)=K, \lim _{t \rightarrow \infty} y(t)=0$; while from the predator equation we see that $\lim _{t \rightarrow \infty} y(t)=+\infty$, a contradiction). From the predator equation, we 
see that, for $t>T$, we have

$$
\begin{aligned}
y^{\prime}(t) & \leqq y\left(-d+\frac{f K}{K+m y}\right) \\
& =y\left(\frac{(f-d) K-d m y(t)}{K+m y}\right) .
\end{aligned}
$$

A standard comparison argument shows that

$$
\lim _{t \rightarrow \infty} \sup y(t) \leqq \frac{f-d}{d m} K .
$$

This shows that system (1.4) is dissipative.

It is easy to see that, for system (1.4), if $m a>c$, then

$$
x^{\prime}>x\left(a-\frac{c}{m}-b x\right)
$$

which implies that $\lim \inf _{t \rightarrow+\infty} x(t) \geqq b^{-1}(a-c / m) \equiv \underline{x}$. Hence, for large $t$, $x(t)>\underline{x} / 2$, and

$$
y^{\prime} \geqq y\left(-d+\frac{f \underline{x} / 2}{m y+\underline{x} / 2}\right) .
$$

We obtain

$$
y^{\prime} \geqq y[(f-d) \underline{x} / 2-d m y(t)] /(m y+\underline{x} / 2)
$$

which yields that for $f>d$,

$$
\lim _{t \rightarrow+\infty} \inf y(t) \geqq(f-d) \underline{x}(2 d m)^{-1} \equiv \underline{y} .
$$

The above arguments imply that:

Theorem 2.2. If $f>d$ and $m a>c$, then system (1.4) is permanent.

Recall that system (1.1) is permanent if and only if it has a positive steady state. The above theorem is not nearly as sharp. The same can be said for most results in the rest of this section.

Assume below that $\mathrm{cm}^{-1}>a+d$ in system (1.4). Then there is an $\alpha>0$, such that $c(m+\alpha)^{-1}=a+d$. Let $\delta=x(0) / y(0)<\alpha$. We claim that for all $t>0, x(t) / y(t)<\alpha$ and $\lim _{t \rightarrow+\infty} x(t)=0$. Otherwise, there is a first time $t_{1}$, $x\left(t_{1}\right) / y\left(t_{1}\right)=\alpha$ and for $t \in\left[0, t_{1}\right), x(t) / y(t)<\alpha$. Then for $t \in\left[0, t_{1}\right]$, we have

$$
\begin{aligned}
x^{\prime}(t) & \leqq x(a-c /(m+x / y)) \leqq x\left(a-\frac{c}{m+\alpha}\right) \\
& =-d x(t)
\end{aligned}
$$


which implies that $x(t) \leqq x(0) \mathrm{e}^{-d t}$. However, for all $t \geqq 0$,

$$
y^{\prime}(t) \geqq-d y(t)
$$

which implies that $y(t) \geqq y(0) \mathrm{e}^{-d t}$. This shows that for $t \in\left[0, t_{1}\right]$,

$$
x(t) / y(t) \leqq x(0) / y(0)=\delta<\alpha,
$$

a contradiction to the existence of $t_{1}$, proving the claim. This in turn implies that $x(t) \leqq x(0) \mathrm{e}^{-d t}$ for all $t \geqq 0$. That is, $\lim _{t \rightarrow+\infty} x(t)=0$. Hence we have established the following result (recall that system (1.4) is said to be not persistent if $\min \left(\lim _{\inf _{t \rightarrow+\infty}} x(t), \lim _{\inf _{t \rightarrow+\infty}} y(t)\right)=0$ for some of its positive solutions):

Theorem 2.3. If $\mathrm{cm}^{-1}>a+d$, then system (1.4) is not persistent.

Note that under the assumption $\mathrm{cm}^{-1}>a+d$, system (1.4) may have positive steady state. This shows that system (1.4) can have both positive steady state and positive solutions that tend to the origin.

In fact, we also have

Theorem 2.4. If $\mathrm{cm}^{-1}>a+d$, then there exist positive solutions $(x(t), y(t))$ of system (1.4) such that $\lim _{t \rightarrow+\infty}(x(t), y(t))=(0,0)$.

Proof. If $f \leqq d$, then this is obvious from the previous argument that leads to Theorem 2.3. Assume now that $f>d$. Again, the argument leading to Theorem 2.3 shows that $\lim _{t \rightarrow+\infty} x(t)=0$ and for $t \geqq 0, x(t) / y(t) \leqq \delta$, provided that $\delta=x(0) / y(0)<\alpha$, where $\alpha=c(d+f)^{-1}-m$. Let $(x(t), y(t))$ be the solution of (1.4), with $x(0) / y(0)<\alpha$. Since $y(t)$ is bounded, we have

$$
0 \leqq s_{1} \equiv \lim _{\sup _{t \rightarrow+\infty}} y(t)<+\infty, \quad 0 \leqq s_{2} \equiv \liminf _{t \rightarrow+\infty} y(t)<+\infty .
$$

If $s_{2}>0$, then we see that for large $t, y^{\prime}(t)<-d y(t) / 2$, which leads to $\lim _{t \rightarrow+\infty} y(t)=0$. This is a contradiction. So we must have $s_{2}=0$. Assume below that $s_{1}>0$. Since $\lim _{t \rightarrow+\infty} x(t)=0$, we see that

(i) there is a $t_{1}$, such that for $t>t_{1}$, we have $x(t)<m d s_{1} /[2(f-d)]$. From the assumption $s_{1}>0$, we see that there is a $t_{2}>t_{1}$ such that $y\left(t_{2}\right)>s_{1} / 2$, and $y^{\prime}\left(t_{2}\right)>0$. However, we note that $y^{\prime}\left(t_{2}\right)>0$ implies

$$
x\left(t_{2}\right)>m d y\left(t_{2}\right) /(f-d)>m d s_{1} /[2(f-d)] .
$$

This is a contradiction to (i). Hence we must have $s_{2}=0$. This completes the proof.

Note that under the assumption $\mathrm{cm}^{-1}>a+d$, system (1.4) may have positive steady state. This shows that system (1.4) can have both positive steady state and positive solutions that tend to the origin. In particular, we see that if $f$ is just slightly greater than $d$ and $\mathrm{cm}^{-1}>a+d$, than $E^{*}$ is locally asymptotically stable but not globally asymptotically stable. 
Theorem 2.4 generalizes theorem 2.6 in Beretta and Kuang (1997). Clearly, Theorem 2.4 implies that if $c>m(a+d)$, the boundary equilibrium $(K, 0)$ of system (1.4) is not globally asymptotically stable. Note that under the condition $c>m(a+d)$, system (1.4) can have no positive steady state and at the same time, $(K, 0)$ is locally stable (just add the assumption $f \leqq d)$. In such case, some solutions tend to $(K, 0)$ and some tend to $(0,0)$. Hence the above theorem shows that system (1.4) can exhibit behavior similar to bistability. However, we can also show that

Theorem 2.5. If $c \leqq m a$ and $f \leqq d$, then $(K, 0)$ is globally asymptotically stable for system (1.4).

Proof. Clearly $\lim _{t \rightarrow+\infty} y(t)=0$, and $\lim \inf _{t \rightarrow+\infty} x(t) \geqq x, x=b^{-1}\left(a-\mathrm{cm}^{-1}\right)$. Assume first that $c<m a$. Then, for any $\varepsilon \in(0, a)$, there exists $T=T(\varepsilon)$, such that for $t>T$,

$$
x(t)(a-\varepsilon-b x(t)) \leqq x^{\prime}(t) \leqq x(t)(a-b x(t)) .
$$

This clearly shows that

$$
\lim _{t \rightarrow+\infty} x(t)=a b^{-1}=K .
$$

Assume now that $c=m a$. Then

$$
x^{\prime}=x^{2}(a-b x-b m y) /(m y+x) .
$$

Since $\lim _{t \rightarrow+\infty} y(t)=0$, we see that (by a standard comparison argument)

$$
\lim _{t \rightarrow+\infty} x(t)=a b^{-1}=K .
$$

This proves the theorem.

Our last theorem of this section shows that under some conditions, the origin can be globally attractive!

Theorem 2.6. If $f \geqq d c /(c-m a)$ and $c>m a$, then $(0,0)$ is globally asymptotically stable for system (1.4).

Proof. This theorem can be proved by a simple flow analysis. Since $f \geqq d c /(c-m a)$ and $c>m a$, we see that the predator isocline lay above the prey isocline. So, we can divide the positive cone into three regions, I, II, III.

$$
\begin{aligned}
I I I & =\{(x, y): x \geqq 0, y \geqq 0, F(x, y) \geqq 0, G(x, y)>0\} ; \\
I I & =\{(x, y): x \geqq 0, y \geqq 0, F(x, y)<0, G(x, y) \geqq 0\} ; \\
I & =\{(x, y): x \geqq 0, y \geqq 0, F(x, y)<0, G(x, y)<0\} .
\end{aligned}
$$

Clearly, solutions that start in region $I I I$ will enter region $I I$ by crossing the prey isocline vertically(upwards) in finite time, since there is no steady state in 
III. And then, for the same reason, the solutions will enter region $I$ in finite time by crossing the predator isocline horizontally (left wards). Once in region $I$, it is easy to see that solutions are trapped (cannot cross the predator isocline from left to right) and we must have $(x(t), y(t)) \rightarrow(0,0)$ as $t \rightarrow+\infty$. This proves the theorem.

\section{Nonexistence of nontrivial positive periodic solutions}

In this section we shall show that if the positive steady state of the system (1.4) is locally asymptotically stable, then the system has no nontrivial positive periodic solutions. Our method involves a nontrivial application of the socalled divergency criterion for the stability of periodic solutions in planar systems (see Hale (1980), Lemma 3.1 on p. 224) and some critical transformations. This is the same method elegantly applied by Butler et al. (1983) on a chemostat model, and their success inspired us.

Obviously, we shall assume that the positive steady state $E^{*}$ exists in the rest of this section. We can rewrite system (1.4) by centering it on the positive equilibrium, i.e., (recall that $b=a / K$ )

$$
\left\{\begin{array}{l}
x^{\prime}=x\left\{-b\left(x-x^{*}\right)+c\left[\left(\frac{y^{*}}{x^{*}}\right) U\left(\frac{x^{*}}{y^{*}}\right)-\left(\frac{y}{x}\right) U\left(\frac{x}{y}\right)\right]\right\} \\
y^{\prime}=f y\left[U\left(\frac{x}{y}\right)-U\left(\frac{x^{*}}{y^{*}}\right)\right],
\end{array}\right.
$$

where $x^{*}, y^{*}>0$ are the components of the positive equilibrium $E^{*}=\left(x^{*}, y^{*}\right)$ and

$$
U(\xi) \equiv \xi /(m+\xi), \quad m>0 .
$$

From now on we denote by

$$
U^{*}=U\left(\frac{x^{*}}{y^{*}}\right)
$$

The structure of (3.1) further suggests the change of variables $(x, y) \rightarrow(x, u=x / y)$, which is not singular in the interior of $R_{+}^{2}$, thus implying that if $(x, u) \rightarrow\left(x^{*}, u^{*}\right)$ then $(x, y) \rightarrow\left(x^{*}, y^{*}\right)$.

Note that:

$$
\begin{aligned}
u^{\prime} & =\left(\frac{x}{y}\right)^{\prime}=\frac{1}{y} x^{\prime}-\frac{x}{y^{2}} y^{\prime} \\
& =\frac{x}{y}\left[-b\left(x-x^{*}\right)+c\left(\frac{U^{*}}{u^{*}}-\frac{U(u)}{u}\right)-f\left(U(u)-U^{*}\right)\right] .
\end{aligned}
$$


According to (3.2), in the variables $(x, u)$, system (3.1) becomes

$$
\left\{\begin{array}{l}
x^{\prime}=x\left[-b\left(x-x^{*}\right)+c\left(\frac{U^{*}}{u^{*}}-\frac{U(u)}{u}\right)\right] \\
u^{\prime}=u\left[-b\left(x-x^{*}\right)+c\left(\frac{U^{*}}{u^{*}}-\frac{U(u)}{u}\right)-f\left(U(u)-U^{*}\right)\right] .
\end{array}\right.
$$

Let us rewrite the term $U^{*} / u^{*}-U / u$ in (3.3) in the following more suitable form:

$$
\frac{U^{*}}{u^{*}}-\frac{U}{u}=\frac{U}{u^{*} u}\left(u-u^{*}\right)-\frac{1}{u^{*}}\left(U(u)-U^{*}\right)
$$

and introduce the following nomenclature. Define by

$$
v_{1}=x-x^{*}, \quad v_{2}=u-u^{*},
$$

such that $v_{1} \geqq-x^{*}, v_{2} \geqq-u^{*}$ and the function:

$$
g\left(v_{2}\right) \equiv U(u)-U^{*}=\frac{m v_{2}}{\left(m+u^{*}\right)(m+u)} .
$$

Observing that:

$$
\begin{gathered}
g\left(v_{2}\right) v_{2} \geqq 0 \quad \text { and }=0 \quad \text { if and only if } v_{2}=0 \\
g^{\prime}\left(v_{2}\right)=\frac{m}{(m+u)^{2}}=\frac{m}{\left(m+u^{*}+v_{2}\right)^{2}} .
\end{gathered}
$$

Let us further remark that, according to $(3.4)-(3.7 \mathrm{a}, \mathrm{b})$,

$$
c\left(\frac{U^{*}}{u^{*}}-\frac{U(u)}{u}\right)=\frac{c U(u)}{u^{*} u} v_{2}-\frac{c g\left(v_{2}\right)}{u^{*}}=\frac{c}{m} g\left(v_{2}\right)
$$

where we used the fact that(from (3.6))

$$
g\left(v_{2}\right)=\frac{m}{m+u^{*}} \cdot \frac{v_{2}}{m+u} .
$$

Hence, from (3.3), we finally get:

$$
\left\{\begin{array}{l}
v_{1}^{\prime}=\left(x^{*}+v_{1}\right)\left[-b v_{1}+\frac{c}{m} g\left(v_{2}\right)\right] \equiv F_{1}\left(v_{1}, v_{2}\right) \\
v_{2}^{\prime}=\left(u^{*}+v_{2}\right)\left[-b v_{1}-\left(f-\frac{c}{m}\right) g\left(v_{2}\right)\right] \equiv F_{2}\left(v_{1}, v_{2}\right) .
\end{array}\right.
$$

Furthermore, note that the positive equilibrium $\left(x^{*}, u^{*}\right)$ in (3.10) corresponds to $v_{1}(t)=v_{2}(t)=0$ for all real value of $t$. Of course, the asymptotic stability of 
the trivial equilibrium of (3.10) implies that of the positive equilibrium of (3.1). Recall that the trace of the Jacobian of system (1.4) at $E^{*}$ is

$\delta^{*} \equiv \operatorname{tr}\left(x^{*}, y^{*}\right) \equiv \frac{\partial F}{\partial x}\left(x^{*}, y^{*}\right)+\frac{\partial G}{\partial y}\left(x^{*}, y^{*}\right)=-b x^{*}+(c-f m) \frac{u^{*}}{\left(m+u^{*}\right)^{2}}$.

Recall also that, $E^{*}$ is locally asymptotically stable if that $\delta^{*}=\operatorname{tr}\left(x^{*}, y^{*}\right)<0$. We shall show below that the same condition assures that the system has no nontrivial positive periodic solutions.

For any positive periodic solution $\Gamma^{\prime}$ of period $T$ that contains $E^{*}$, we have a corresponding positive periodic solution $\Gamma$ in $\left(v_{1}, v_{2}\right)$ plane that contains $(0,0)$. Clearly, $\Gamma$ is orbitally stable if and only if $\Gamma^{\prime}$ is so. Note, however, as $t$ increases, $(x(t), y(t))$ travels $\Gamma^{\prime}$ anti clockwise (has positive orientation), while $\left(v(t), v_{2}(t)\right)$ travels clockwise (has negative orientation). we set

$$
\Delta(\Gamma) \equiv \int_{0}^{T}\left(\frac{\partial F_{1}}{\partial v_{1}}+\frac{\partial F_{2}}{\partial v_{2}}\right) d t
$$

Recall that $\Delta(\Gamma)<0$ implies that $\Gamma$ is asymptotically (orbitally) stable (see Lemma 3.1, p. 224 in Hale (1980).

Now we are in a position to state the main results of this section.

Theorem 3.1. If the positive steady state of the system (1.4) is locally asymptotically stable, then the system has no nontrivial positive periodic solutions. In other words, positive solutions either tend to the origin or to $E^{*}$.

Our method of proving the above theorem is to show that if $E^{*}$ is locally stable and there is a positive periodic solution $\Gamma$ surrounding it, then $\Delta(\Gamma)<0$. That is all positive periodic solutions that surround $E^{*}$, if exist, must be orbitally stable. This, however, is impossible, since $E^{*}$ is also asymptotically stable. Therefore, system (1.4) cannot have any positive periodic solution (all positive periodic solutions have to contain $E^{*}$ in their interior), and hence positive solutions either tend to the origin or to $E^{*}$.

The proof of the above theorem follows directly from the next two lemmas.

Lemma 3.1. In system (1.4), assume that $\delta^{*}=\operatorname{tr}\left(x^{*}, y^{*}\right)<0$ and $c \leqq f m$, then $\Delta(\Gamma)<0$ for all positive periodic solutions that surround $E^{*}$.

Proof. We note that $x=x^{*}+v_{1}, u=u^{*}+v_{2}$. Hence $v_{1}{ }^{\prime}=x^{\prime}=F_{1}$, $v_{2}{ }^{\prime}=u^{\prime}=F_{2}$. For any positive periodic solution $\Gamma$ that surrounds $E^{*}$, we thus have

$$
\int_{0}^{T} \frac{F_{1}}{x^{*}+v_{1}} d t=\int_{0}^{T} d(\ln x)=0, \int_{0}^{T} \frac{F_{2}}{u^{*}+v_{2}} d t=\int_{0}^{T} d(\ln u)=0 .
$$

Therefore

$$
\Delta(\Gamma)=\int_{0}^{T}\left\{-b\left(x^{*}+v_{1}\right)-\left(u^{*}+v_{2}\right)(f-c / m) g^{\prime}\left(v_{2}\right)\right\} d t .
$$


After trivial simplifications, we have

$$
\Delta(\Gamma)=\int_{0}^{T}\left\{-b x+(c-f m) u /(m+u)^{2}\right\} d t .
$$

Since $x=x^{*}+v_{1}>0, u=u^{*}+v_{2}>0$, we see that if $c \leqq f m$, then $\Delta(\Gamma)<0$. This proves the lemma.

Next lemma shows that even if $c>f m$, we still have $\Delta(\Gamma)<0$.

Lemma 3.2. In system (1.4), assume that $\delta^{*}=\operatorname{tr}\left(x^{*}, y^{*}\right)<0$ and $c>f m$, then $\Delta(\Gamma)<0$ for all positive periodic solutions that surround $E^{*}$.

Proof. First, we observe that

$$
v_{1}^{\prime} / x-v_{2}^{\prime} / u=f g\left(v_{2}\right) .
$$

From which we obtain

$$
-b v_{1}=(1-c /(m f)) v_{1}^{\prime} / x+(c / m f) v_{2}^{\prime} / u .
$$

This together with eq (3.13) imply that

$$
\int_{0}^{T}-b v_{1} d t=0
$$

Hence we obtain

$$
\begin{aligned}
\Delta(\Gamma) & =-b x^{*} T+\int_{0}^{T} \frac{(c-f m) u}{(m+u)^{2}} d t \\
& =\delta^{*} T+\int_{0}^{T}(c-f m)\left[\frac{u}{(m+u)^{2}}-\frac{u^{*}}{\left(m+u^{*}\right)^{2}}\right] d t .
\end{aligned}
$$

Note that

$$
\frac{u}{(m+u)^{2}}-\frac{u^{*}}{\left(m+u^{*}\right)^{2}}=\frac{\left(m+u^{*}\right)^{2}\left(u^{*}+v_{2}\right)-u^{*}\left(m+u^{*}+v_{2}\right)^{2}}{(m+u)^{2}\left(m+u^{*}\right)^{2}}
$$

and

$\left(m+u^{*}\right)^{2}\left(u^{*}+v_{2}\right)-u^{*}\left(m+u^{*}+v_{2}\right)^{2}=\left(m+u^{*}\right)^{2} v_{2}-u^{*} v_{2}^{2}-2 u^{*} v_{2}\left(m+u^{*}\right)$.

We thus have (recall that $\left.g=g\left(v_{2}\right)=m v_{2} /\left[(m+u)\left(m+u^{*}\right)\right]=f^{-1}\left(v_{1}^{\prime} / x-v_{2}^{\prime} / u\right)\right)$

$$
\begin{aligned}
\frac{u}{(m+u)^{2}}-\frac{u^{*}}{\left(m+u^{*}\right)^{2}} & =\frac{\left(m+u^{*}\right)}{m(m+u)} g-\frac{u^{*}}{m^{2}} g^{2}-\frac{2 u^{*}}{m(m+u)} g \\
& =g\left(\frac{\left(m-u^{*}\right)}{m(m+u)}-\frac{u^{*}}{m^{2}} g\right) .
\end{aligned}
$$

For convenience, we define $D\left(v_{2}\right)$ by

$$
D\left(v_{2}\right)=\frac{\left(m-u^{*}\right)}{m(m+u)}-\frac{u^{*}}{m^{2}} g .
$$


Hence, we have

$$
\frac{u}{(m+u)^{2}}-\frac{u^{*}}{\left(m+u^{*}\right)^{2}}=f^{-1}\left(\frac{v_{1}^{\prime}}{x}-\frac{v_{2}^{\prime}}{u}\right) D\left(v_{2}\right) .
$$

Therefore, we have

$$
\Delta(\Gamma)=\delta^{*} T+\frac{c-f m}{f} \int_{0}^{T}\left(\frac{v_{1}^{\prime}}{x} D\left(v_{2}\right)-\frac{v_{2}^{\prime}}{u} D\left(v_{2}\right)\right) d t .
$$

By converting the time integral to line integral, we obtain

$$
\Delta(\Gamma)=\delta^{*} T+\frac{c-f m}{f} \oint_{\Gamma}\left(\frac{D\left(v_{2}\right)}{x^{*}+v_{1}} d v_{1}-\frac{D\left(v_{2}\right)}{u^{*}+v_{2}} d v_{2}\right) .
$$

By applying Green theorem to the line integral, we obtain (be careful about the sign change due to the negative orientation of $\Gamma$ )

$$
\begin{aligned}
\oint_{\Gamma}\left(\frac{D\left(v_{2}\right) d v_{1}}{x^{*}+v_{1}}-\frac{D\left(v_{2}\right) d v_{2}}{u^{*}+v_{2}}\right) & =-\oint_{-\Gamma}\left(\frac{D\left(v_{2}\right) d v_{1}}{x^{*}+v_{1}}-\frac{D\left(v_{2}\right) d v_{2}}{u^{*}+v_{2}}\right) \\
& =\iint_{\mathrm{A}} \frac{\partial}{\partial v_{2}}\left(\frac{D\left(v_{2}\right)}{x^{*}+v_{1}}\right) d v_{1} d v_{2}
\end{aligned}
$$

Where $A$ is the region bounded by $\Gamma$. A straightforward computation shows that

$$
\frac{\partial}{\partial v_{2}}\left(\frac{D\left(v_{2}\right)}{x^{*}+v_{1}}\right)=-\frac{1}{\left(x^{*}+v_{1}\right)(m+u)^{2}}<0 .
$$

Hence we have

$$
\Delta(\Gamma)=\delta^{*} T-\frac{c-f m}{f} \iint_{A} \frac{d v_{1} d v_{2}}{\left(x^{*}+v_{1}\right)(m+u)^{2}} .
$$

Therefore, we have shown that if $\delta^{*}<0$, then so must be $\Delta(\Gamma)$. This completes the proof.

Now, we are ready to state the proof of our main theorem.

Proof of Theorem 3.1. The remark made right after the statement of the theorem together with the above two lemmas imply that under the assumption of the theorem, system (1.4) cannot have positive periodic solutions. The dissipativity of the system together with the saddle property of $(K, 0)$ (and Poincare-Bendixon theorem) imply that positive solutions of system (1.4) tend to either the origin or to $E^{*}$.

Corollary 3.1. In system (1.4), assume that $\delta^{*}=\operatorname{tr}\left(x^{*}, y^{*}\right)<0$ and $c \leqq m a$, then $E^{*}$ is globally asymptotically stable.

Proof. If $c<m a$, then by theorem 2.2, we see that positive solutions can not tend to the origin (in fact, not to the boundary). Hence, positive solutions must 
tend to $E^{*}$. If $c=m a$, then we have

$$
x^{\prime}=x^{2}(-b+a /(m y+x)) .
$$

From this equation, we see that if

$$
\lim _{t \rightarrow+\infty}(x(t), y(t))=(0,0),
$$

then we see that for large $t$, we have $a /(m y+x)$ much larger than $b$. Hence for large $t$, we have $x^{\prime}(t)>0$. This is a contradiction. Therefore, positive solutions cannot tend to the origin, instead, they must tend to $E^{*}$.

To end this section, we would like to include the following global stability result from Beretta and Kuang (1997) (Corollary 3.5). Its proof involves the construction of a proper Liapunov function. It can be shown that if $c>m a$ and $c<m f$, then $f<d c /(c-m a)$ implies that $c m^{-1}<a+d$. In such case, Theorem 2.4 does not apply.

Theorem 3.2. Assume that the positive equilibrium of $\left(x^{*}, y^{*}\right)$ exists and that $\mathrm{fm}-\mathrm{c}>0$. Then it is globally asymptotically stable.

\section{Discussion}

In this paper, we have shown that compared to the traditional predator-prey system (1.1), the ratio-dependent predator-prey system (1.4) has much richer boundary dynamics. For ratio-dependent predator-prey models, there can be no paradox of enrichment or paradox of biological control. Even when there is no positive steady state, both prey and predator can become extinct. Such extinction occurs in two cases. In one case, both species become extinct regardless of the initial data. In the other case, both species will die out only if initial prey/predator ratio is too low. Otherwise, only the predator goes extinct, while prey tends to carrying capacity. These are all clearly reasonable outcomes that are frequently observed (Getz (1984)) in the nature. However, traditional predator-prey systems fail to exhibit these.

While the above facts do not provide enough evidences to discredit the traditional predator-prey models, it suggests the traditional predator-prey models have a number of limitations. Our conviction is that the ratio-dependent predator-prey models are more appropriate for predator-prey interactions where predation involve serious searching processes (like animals hunting for animals), while the traditional ones may be more suitable to those involve no or random searching process (like chemostat situation, plantherbivore interaction, etc.).

Notice there are six parameters in (1.4) or (2.1). If we nondimensionalize the system (1.4) with the substitutions such as

$$
t \rightarrow a t, \quad x \rightarrow x / K, \quad y \rightarrow \frac{m}{K} y
$$


then the system takes the following simpler or neater form

$$
\begin{aligned}
& x^{\prime}=x(1-x)-\frac{s x y}{x+y} \\
& y^{\prime}=\delta y\left[\frac{x}{x+y}-r\right]
\end{aligned}
$$

with three parameters

$$
s=\frac{c}{m a}, \quad \delta=\frac{f}{a}, \quad r=\frac{d}{f} .
$$

With these parameters $(s, \delta, r)$, the stability condition for $E^{*}, \operatorname{tr}\left(x^{*}, y^{*}\right)<0$ becomes

$$
0<s<\frac{r \delta}{1+r}+\frac{1}{1-r^{2}}=\frac{1}{1-r} \frac{r \delta(1-r)+1}{1+r} .
$$

The other conditions appearing in the Theorems can be listed as following

$$
\begin{array}{lll}
f>d & \text { if and only if } & 0<r<1 \\
f<d c /(c-m a) & \text { if and only if } & 1<s<\frac{1}{1-r} \\
c>m a & \text { if and only if } & s>1 \\
c m^{-1}>a+d & \text { if and only if } & s>1+\delta r .
\end{array}
$$

For convenience, we list the results of the previous sections and some questions in terms of nondimensionalized parameters $s, \delta, r$ in Table 1. In the table, locally stable and globally stable refers to locally asymptotically stable and globally asymptotically stable, respectively.

We note from the table that in item 7 and 8 , we have

$$
\frac{r \delta}{1+r}+\frac{1}{1-r^{2}}<1+\delta r \text { if and only if } \delta(1-r)>1 .
$$

For 7 and 8 (i), our simulation work leads us to conjecture that $E^{*}$ is in fact globally stable. In the case of 8 (ii), as we expected, some solutions tend to $E^{*}$, while others go to the origin. For the case 8(iii), our numerical work clearly shows that when the value of $c$ is increased to slightly exceed the bifurcation value, a stable limit cycle bifurcates from the unstable positive steady state $E^{*}$. The size of this limit cycle increases rapidly and may disappear as $c$ is further increased, resulting in some or all positive solutions (except $E^{*}$ ) being attracted by the origin $(0,0)$.

An obvious yet interesting biological implication of condition 7 (iii) of Table 1 is that when $E^{*}$ is unstable, then extinction of both species is always a possibility. To avoid extinction of either species, it is highly desirable to have 
Table 1. Stability results and questions in terms of $s, \delta, r$

Conditions Results or question

1. $r \geqq 1, s>0$,

2. $r \geqq 1,0<s \leqq 1$,

3. $s>1+\delta r$,

4. $r \geqq 1,1<s \leqq 1+\delta r$,

5. $0<r<1,0<s \leqq 1$,

6. $0<r<1, s>1 /(1-r)$,

7. $0<r<1,1<s<1 /(1-r)$ $\delta(1-r) \geqq 1$

8. $0<r<1,1<s<1 /(1-r)$ $\delta(1-r)<1$
$(1,0)$ is locally asymptotically stable (by a standard comparison argument)

$(1,0)$ is globally asymptotically stable (Theorem 2.5)

There exists $(x(t), y(t)) \rightarrow(0,0)$ as $t \rightarrow \infty$ Hence, the system is not persistent

(Theorem 2.4)

$(1,0)$ is locally stable

QUESTION: Is $(1,0)$ globally stable?

$E^{*}$ is globally stable (Corollary 3.1 )

$(0,0)$ is globally stable (Theorem 2.6 )

$E^{*}$ is locally stable

QUESTION: Is $E^{*}$ globally stable?

(i) QUESTION: Is it true that if $1<s \leqq 1+\delta r$, then $E^{*}$ is globally stable?

(ii) If $1+\delta r<s<1 /(1-r)^{2}+r \delta /(1+r), E^{*}$ is locally stable and the system is not persistent

(iii) $1 /(1-r)^{2}+r \delta /(1+r)<s<1 /(1-r)$ then $E^{*}$ is unstable and the system is not persistent.

parameters satisfy condition 4 of Table 1, or, possibly, condition 6 or condition 7 (i) of Table 1.

We note also that if we set $u=x / y$, then the system becomes

$$
\begin{aligned}
& x^{\prime}=x(1-x)-\frac{s x}{1+u}, \\
& u^{\prime}=u\left[(1-x)-\frac{s}{1+u}-\delta\left(\frac{u}{1+u}-r\right)\right] .
\end{aligned}
$$

This system has no singular point, thus local stability of all nonnegative steady states can be studied.

There are still many interesting and challenging mathematical questions need to be studied for system (1.4), and a lot more for system (1.2). For example, most of our results in Sect. 2 have room for improvement. However, significant improvements appear to be difficult. Also, we are unable to show system (1.4) has unique positive limit cycle, when $E^{*}$ exists and is unstable. This is known to be true for system (1.1) (see Kuang and Freedman (1988) and the reference cited).

A hybrid type of the traditional and ratio-dependent predator-prey model is the so-called Holling-Tanner (May (1974)) predator-prey model which 
takes the form of

$$
\left\{\begin{array}{l}
x^{\prime}=\operatorname{ax}(1-x / K)-c x y /(m+x) \\
y^{\prime}=d y(1-f y / x) .
\end{array}\right.
$$

Here $a, K, c, m, d, f$ are positive constants whose biological meaning are obvious. The basic assumption here is that if the prey density $x$ is constant, then it provides a carrying capacity of $x / f$ for the predator. Clearly, the HT (Holling-Tanner) model is somewhat related to ratio-dependent one in predator equation. While it has a prey equation of the classic Lotka-Volterra type. This model has been systematically studied in the recent work by Hsu and Huang (1995).

Finally, we would like to point out that there is almost nothing has been done to the qualitative analyses of food chains or multi-species interaction models based on ratio-dependent approach. It is expected, such models are richer in dynamics than the traditional counterparts. And most surely, they pose more interesting and challenging questions both mathematically and biologically.

Acknowledgement. The authors would like to thank the referee for helpful suggestions including the construction of Table 1 .

\section{References}

Arditi, R., Berryman, A. A.: The biological control paradox. Trends in Ecology and Evolution 6, 32 (1991)

Arditi, R., Ginzburg, L. R.: Coupling in predator-prey dynamics: ratio-dependence. J. Theoretical Biology 139, 311-326 (1989)

Arditi, R., Ginzburg, L. R., Akcakaya, H. R.: Variation in plankton densities among lakes: a case for ratio-dependent models. American Naturalist 138, 1287-1296 (1991)

Arditi, R., Perrin, N., Saiah, H.: Functional responses and heterogeneities: an experimental test with cladocerans, OIKOS 60, 69-75 (1991)

Arditi, R., Saiah, H.: Empirical evidence of the role of heterogeneity in ratio-dependent consumption, Ecology 73, 1544-1551 (1992)

Berryman, A. A.: The origins and evolution of predator-prey theory, Ecology 73, $1530-1535$ (1992)

Beretta, E., Kuang, Y.: Convergence results in a well known delayed predator-prey system, J. Math. Anal. Appl. 204, 840-853 (1996)

Beretta, E., Kuang, Y.: Global analyses in some delayed ratio-dependent predator-prey systems, Nonlinear Analysis, TMA., (1997), in press

Butler, G. J., Hsu, S. B., Waltman, P.: Coexistence of competing predators in a chemostat, J. Math. Biol. 17, 133-151 (1983)

Freedman, H. I.: Deterministic Mathematical Models in Population Ecology, Marcel Dekker, New York, 1980

Getz, W. M.: Population dynamics: a per-capita resource approach, J. Theoretical Biology 108, 623-643 (1984)

Ginzburg, L. R., Akcakaya, H. R.: Consequences of ratio-dependent predation for steady state properties of ecosystems, Ecology 73, 1536-1543 (1992)

Gutierrez, A. P.: The physiological basis of ratio-dependent predator-prey theory: a metabolic pool model of Nicholson's blowflies as an example, Ecology 73, 1552-1563 (1992) 
Hairston, N. G., Smith, F. E., Slobodkin, L. B.: Community structure, population control and competition, American Naturalist 94, 421-425 (1960)

Hale, J. K.: Ordinary Differential Equations, Krieger Publishing Co. Malabar, 1980

Hanski, I.: The functional response of predator: worries about scale, TREE 6, 141-142 (1991)

Hsu, S.-B., Huang, T.-W.: Global stability for a class of predator-prey systems, SIAM J. Appl. Math., 55, 763-783 (1995)

Kuang, Y.: Delay Differential Equations with Applications in Population Dynamics, vol. 191 in the series of Mathematics in Science and Engineering, Academic Press, Boston, 1993

Kuang, Y.: Global stability in delay differential systems without dominating instantaneous negative feedbacks, J. Diff. Eqns. 119, 503-532 (1995)

Kuang, Y.: Nonuniqueness of limit cycles of Gause-type predator-prey systems, Appl. Anal. 29, 269-287 (1988)

Kuang, Y.: Global stability of Gause-type predator-prey systems, J. Math. Biol. 28, 463-474 (1990)

Kuang, Y., Beretta, E.: Global analysis of Gause-type ratio-dependent predator-prey systems, (1997), submitted

Kuang, Y., Freedman, H. I.: Uniqueness of limit cycles in Gause-type predator-prey systems, Math. Biosci. 88, 67-84 (1988)

Luck, R. F.: Evaluation of natural enemies for biological control: a behavior approach, Trends in Ecology and Evolution 5, 196-199 (1990)

May, R. M.: Stability and Complexity in Model Ecosystems, Princeton Univ. Press, 1974

Maynard Smith, J.: Models in Ecology, Cambridge Univ. Press, 1974

Rosenzweig, M. L.: Paradox of enrichment: destabilization of exploitation systems in ecological time, Science 171, 385-387 (1969) 